\title{
1. Dynamic estimation of three-dimensional cerebrovascular deformation from 2 rotational angiography
}

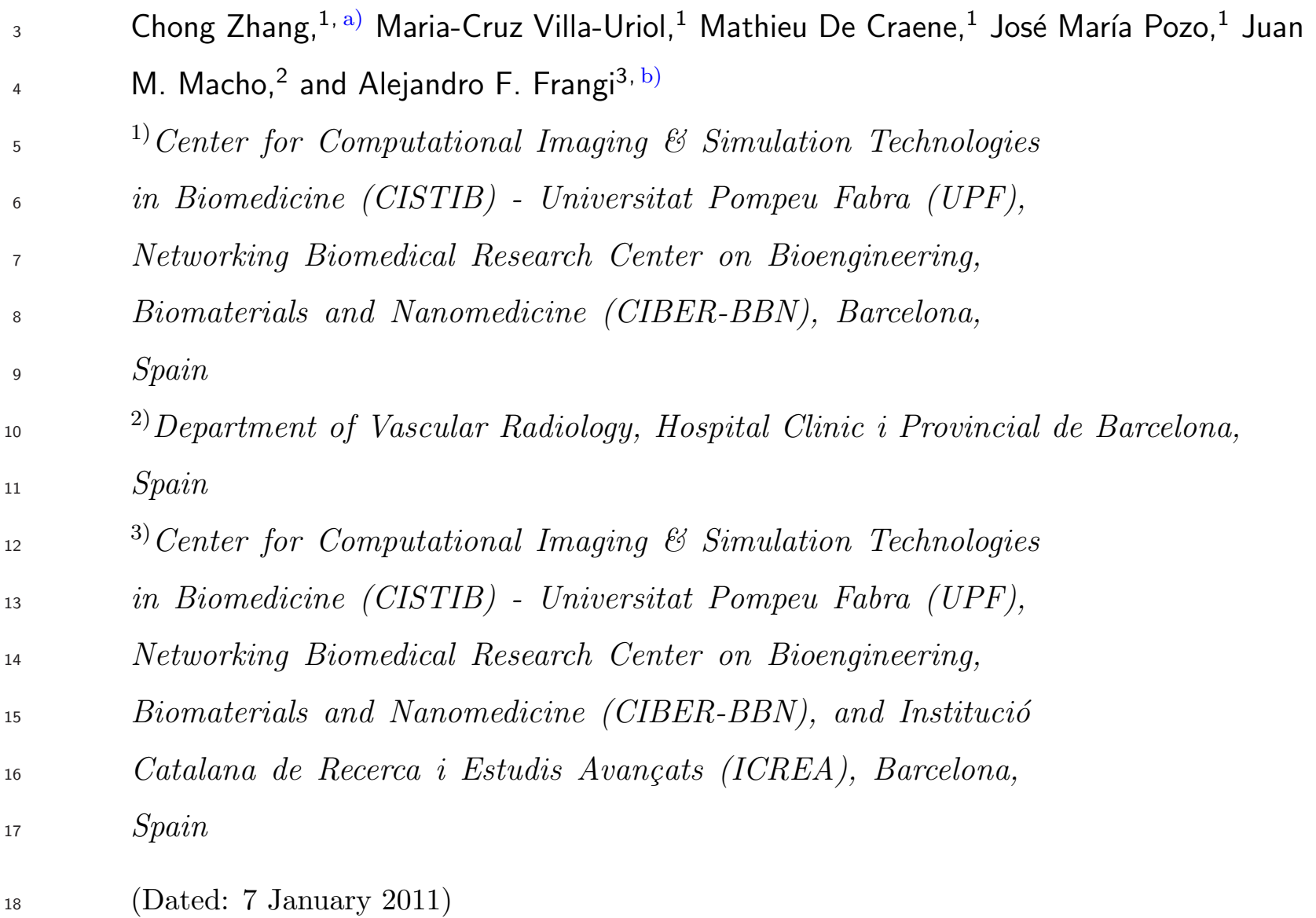


Purpose: The objective of this study is to investigate the feasibility of detecting and quantifying 3D cerebrovascular wall motion from a single 3D rotational X-ray angiography (3DRA) acquisition within a clinically acceptable time, and computing from the estimated motion field for the further biomechanical modeling of the cerebrovascular wall.

Methods: The whole motion cycle of the cerebral vasculature is modeled using a 4D $B$-spline transformation, which is estimated from a $4 \mathrm{D}$ to $2 \mathrm{D}+\mathrm{t}$ image registration framework. The registration is performed by optimizing a single similarity metric between the entire $2 \mathrm{D}+\mathrm{t}$ measured projection sequence and the corresponding forward projections of the deformed volume at their exact time instants. The joint use of two acceleration strategies together with their implementation on graphics processing units are also proposed so as to reach computation times close to clinical requirements. For further characterizing vessel wall properties, an approximation of the wall thickness changes is obtained through a strain calculation.

Results: Evaluation on in silico and in vitro pulsating phantom aneurysms demonstrated an accurate estimation of wall motion curves. In general, the error was below $10 \%$ of the maximum pulsation, even in the situation when substantial inhomogeneous intensity pattern was present. Experiments on in vivo data provided realistic aneurysm and vessel wall motion estimates, whereas in regions where motion was neither visible nor anatomically possible no motion was detected. The use of the acceleration strategies enabled completing the estimation process for one entire cycle in 5-10 minutes without degrading the overall performance. The strain map extracted from our motion estimation provided a realistic deformation measure of the vessel wall.

Conclusions: Our technique has demonstrated that it can provide accurate and robust 4D estimates of cerebrovascular wall motion within a clinically acceptable time, although it has to be applied to a larger patient population prior to possible wide application to routine endovascular procedures. In particular, 
for the first time, this feasibility study has shown that in vivo cerebrovascular motion can be obtained intra-procedurally from a 3DRA acquisition. Results have also shown the potential of performing strain analysis using this imaging modality, making thus possible for the future modeling of biomechanical properties of the vascular wall.

19 Keywords: cerebral vasculature; image registration; motion estimation; rotational angiography

${ }^{a)}$ Electronic mail: chong.zhang@upf.edu

b) Electronic mail: alejandro.frangi@upf.edu 


\section{${ }_{21}$ I. INTRODUCTION}

22 Cerebrovascular diseases in general cause changes to the architecture of blood vessels in 23 the brain by making them narrow, stiff, deformed, or uneven. The pathogenesis of these ${ }_{24}$ diseases is believed to be dependent on the complex interactions among multiple physiologi25 cal and mechanical factors such as hemodynamics, wall biomechanics and mechanobiology ${ }^{1}$. ${ }_{26}$ Unfortunately, patient-specific vessel wall properties cannot be measured in vivo with cur${ }_{27}$ rent medical imaging techniques ${ }^{2}$. In many situations, an inverse problem approach based 28 on a mathematical model for the biomechanics of the vasculature is a valid surrogate to 29 estimate material and structural parameters ${ }^{3,4}$. An example of such approach consists of so determining these unknown parameters by applying known boundary conditions on the ves31 sel wall and analyzing its mechanical responses such as vascular wall motion. Tracking this 32 motion should also allow embedding wall compliance as a boundary condition for hemo${ }_{33}$ dynamic simulations ${ }^{5}$. Besides, other studies suggest that even the direct visualization of 34 wall motion abnormalities may be helpful for analyzing pathological features of the cerebral 35 vasculature ${ }^{6,7}$. Therefore, quantifying vascular wall motion and deformation has the poten36 tial of impacting treatment selection and preoperative planning of cerebrovascular diseases. ${ }_{37}$ However, since such motion is in general expected to be in a sub-millimeter range ${ }^{7-9}$, it 38 represents a challenge in terms of the available image resolution of current clinical imaging 39 techniques.

40 Various techniques have been proposed for estimating motion or reconstructing dynamic ${ }_{41} 3 \mathrm{D}$ structures using projection images acquired from image modalities like three-dimensional 42 rotational X-ray angiography (3DRA) and cone beam computed tomography (CBCT). ECG${ }_{43}$ gated techniques ${ }^{10-12}$ constitute the most typical approach, where a reduced set of projections 44 linked to a particular cardiac phase is used to reconstruct a volumetric image using itera${ }_{45}$ tive $^{13}$ or analytical ${ }^{11,14}$ reconstruction methods. Recently, a technique ${ }^{15}$ has been proposed 46 to incorporate a $4 \mathrm{D}$ motion estimation into a projection motion-compensated 3D recon47 struction process by comparing the latter to an initial reference reconstruction. However, ${ }_{48}$ the estimated motion could be limited by the 3D reconstruction error even before performing ${ }_{49}$ the $3 \mathrm{D} / 3 \mathrm{D}$ registration. In other works ${ }^{16,17}$, continuous respiratory motion during a CBCT 50 acquisition has been estimated by optimizing the similarity between the measured and the ${ }_{51}$ corresponding views of a deforming reference volume obtained from CT. However, their tech- 
${ }_{52}$ niques need additional motion constraints such as a prior motion model or a regularization 53 term. Also, their need of two acquisitions increases patient exposure to radiation, limiting 54 their clinical applicability.

55 We aim to retrieve the dynamic 3D morphology of a structure of interest from a sin${ }_{56}$ gle 3DRA acquisition (e.g. cerebral aneurysm or a vessel segment). 3DRA is routinely ${ }_{57}$ performed in clinical practice during endovascular interventions. One standard acquisition ${ }_{58}$ provides a sequence of $2 \mathrm{D}$ rotational X-ray angiographies and an isotropic high-resolution ${ }_{59} 3 \mathrm{D}$ volumetric image reconstructed from them. A physiological signal synchronized with ${ }_{60}$ the projections can also be recorded. In a previous work ${ }^{18}$, we proposed a method to esti${ }_{61}$ mate the 3D morphology of the structure of interest at a given time instant by registering ${ }_{62}$ forward projections of the deformed 3DRA volume to a sparse set of 2D measured projec${ }_{63}$ tions through a temporal weighting scheme. However, since this technique only represents ${ }_{64}$ the spatiotemporal motion through independent 3D morphology estimation at discrete time ${ }_{65}$ points, it fails to address the intrinsic temporal consistency or continuity of motion. In ad${ }_{66}$ dition, the estimated morphology can be compromised by the residual motion introduced by ${ }_{67}$ forcing the forward projections at a specific time instant to match the measured projections ${ }_{68}$ in its temporal vicinity. In general, this problem is also common for ECG-gated methods.

${ }_{69}$ In this paper, instead of representing the motion over time by independent 3D trans70 formations as proposed in $^{18}$, we employ a single $4 \mathrm{D} B$-spline transformation model for the 71 whole motion cycle. It is estimated from a $4 \mathrm{D}$ to $2 \mathrm{D}+\mathrm{t}$ image registration framework. The 72 basic idea of the transformation model is to deform an object by manipulating an underlying 73 mesh of control points, resulting in a smooth and continuous deformation of the reference 74 image at any time of the motion cycle. Thus, an estimate of arbitrarily small displace75 ment or deformation can be achieved through the interpolation from the movements of the 76 control points. Meanwhile, the registration is optimized by measuring a single similarity 77 metric between the entire measured projection sequence and the corresponding forward pro78 jections of the deformed volume at their corresponding exact time instants. This improves 79 the temporal consistency without introducing blurring, as well as the robustness to image 80 noise and artifacts such as contrast agent induced intensity inhomogeneity. Performing the ${ }_{81}$ motion estimation from the projection space improves the accuracy of the motion estimate ${ }_{82}$ as the pixel resolution is higher in the $2 \mathrm{D}+\mathrm{t}$ measured projections than in the $3 \mathrm{D}$ image. ${ }_{83}$ On the other hand, computational cost is high for the simultaneous processing of such high- 
${ }_{84}$ resolution temporal sequences of 3D images, 2D measured and forward projections. We 85 therefore introduce the joint use of two acceleration strategies: a precomputation at the 86 forward projection generation stage and an object-adaptive region-of-interest (ROI) for the 87 forward projection update and the metric computation. Since less data have to be processed, 88 these strategies also result in a reduction of memory requirements. Preliminary results and ${ }_{89}$ the overall registration framework were previously published as in ${ }^{19}$. Here a detailed method 90 description is presented, with the integration of the acceleration strategies implemented on ${ }_{91}$ graphics processing units $(\mathrm{GPU})^{20}$. An extended validation is performed on in silico, in vitro 92 phantoms, and for the first time, on in vivo patient data. In this paper, we also explore 93 whether strain as estimated from the motion field from imaging data can be applied to the ${ }_{94}$ personalization of modeling of the vascular wall biomechanical properties.

\section{${ }_{95}$ II. MATERIAL AND METHODS}

\section{${ }_{96}$ II.A. Motion estimation algorithm}

97 The motion estimation algorithm presented in this paper consists of three steps. First, in 98 order to overcome the limited spatial coverage from each of the separate motion cycles, the 99 measured projections are reordered and built into one canonical motion cycle, according to 100 a synchronized physiological signal such as ECG. Second, a 4D-to-2D+t image registration 101 is performed to obtain a single spatiotemporal transformation field over the whole canonical 102 motion cycle. Third, after obtaining the optimal transformation parameter, instantaneous ${ }_{103} 3 \mathrm{D}$ images of the analyzed morphology at any desired time instant can be extracted by 104 applying the 4D transformation to the reference volume image.

\section{${ }_{105}$ II.A.1. Canonical motion cycle}

106 During the rotational run, the total angular coverage of the measured projections for one 107 cardiac or motion cycle is $40-50^{\circ}$. Such viewing range may not be informative about the ${ }_{108} 3 \mathrm{D}$ motion along certain directions. This drawback could be potentially compensated for by 109 providing an a priori motion model as $i^{17}$. An alternative is to add a pseudo-periodicity 110 constraint term to the optimization function as $\mathrm{in}^{16}$. However, the optimization process 111 is complicated by the need of determining empirically the weight for such regularization. 


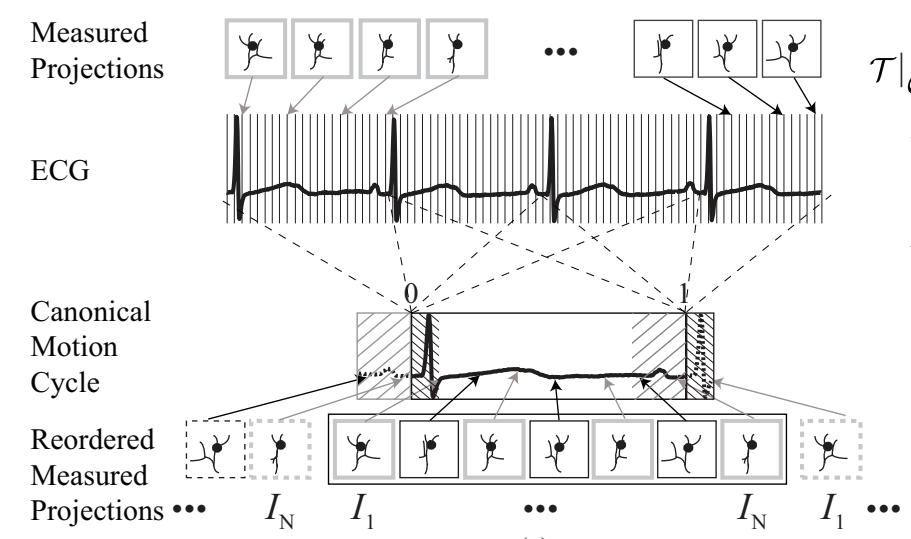

(a)

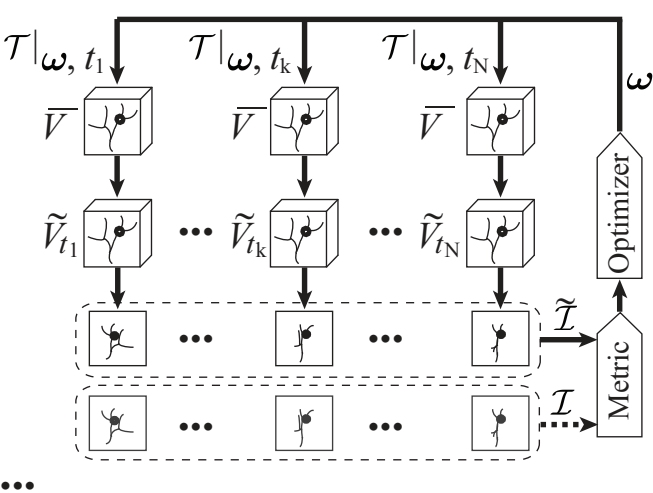

(b)

FIG. 1. (a) Each motion cycle in the $N$ measured projections sequence is normalized to have a unitary duration, according to a physiological signal such as ECG. And the time for each projection is normalized to the full length of its corresponding cardiac cycle. Thus all projections fall within a $[0,1)$ interval. A reordered measured projections sequence can then be obtained based on this normalized time to form one canonical motion cycle. (b) An overview of the $4 \mathrm{D}$-to-2D+t image registration framework, where one metric measuring the similarity between the measured and forward projection sequences, $\mathcal{I}$ and $\tilde{\mathcal{I}}$, is used to estimate a $4 \mathrm{D}$ continuous and smooth transformation model parameterized according to $\boldsymbol{\omega}$ over space and time.

112 We overcome this limitation by reordering all the projections (spanning 4-5 cardiac cycles) 113 to build one canonical motion cycle. This step is carried out as described in ${ }^{18}$ and as 114 illustrated in Fig. 1(a). We first normalize the period of each cycle to have a unitary duration 115 according to a physiological signal such as ECG, which is recorded synchronously together 116 with the projections. The time for each of the $N$ measured projections is normalized to the 117 full length of its corresponding cardiac cycle. Hence, all projections fall within the $[0,1)$ 118 interval and are then sorted by this normalized time to build one canonical motion cycle 119 as $\mathcal{I}=\left\{I_{t_{k}}(\mathbf{x}) \mid k=1 \ldots N\right\}$, where $I_{t_{k}}(\mathbf{x})$ represents the measured projection and at the 120 normalized time $t_{k}\left(0 \leq t_{k} \leq t_{k+1}<1\right)$. In practice, images acquired at similar cardiac 121 phases in the canonical cycle are approximately separated by a $40-50^{\circ}$ angular shift per 122 cycle. By the use of this compounding strategy, the projection spatial viewing angle range 123 is enriched at any temporal vicinity. In addition, the temporal resolution can be considered 124 to be approximately increased by a factor corresponding to the number of cycles during the 125 acquisition. 


\section{II.A.2. 4D-to-2D+t image registration}

${ }_{127}$ The entire measured projection sequence is simultaneously processed to estimate a $4 \mathrm{D}$ 128 continuous and smooth transformation model parameterized over space and time. A single 129 metric captures the similarity between projection sequences instead of considering separate 130 similarities between individual projections.

131 As shown in Fig. 1(b), motion throughout the canonical cycle is represented by a trans${ }_{132}$ formation $\mathcal{T}$ parameterized by $\boldsymbol{\omega}$. Thus, the $3 \mathrm{D}$ instantaneous motion at time $t$ is given by 133 deforming a reference volumetric image $\bar{V}$ :

$$
\widetilde{V}_{t}(\mathbf{p})=\bar{V}(\mathcal{T}(\boldsymbol{\omega}, \mathbf{p}, t))
$$

${ }_{134}$ where $\mathbf{p}$ is a point in $\widetilde{V}_{t}$. In this paper, a $B$-spline based transformation ${ }^{21,22}$ is used. The 135 displacement of $\mathbf{p}$ is represented by a $4 \mathrm{D}$ tensor product of cubic $B$-spline functions (i.e. ${ }_{136} \beta(\cdot)$ in the temporal dimension and $B(\cdot)$ the 3D tensor of $\beta(\cdot)$ in the spatial dimensions), 137 defined on a sparse control points grid $\left(\mathbf{p}_{\mathbf{c}}, t_{\tau}\right)$ :

$$
\mathcal{T}(\boldsymbol{\omega}, \mathbf{p}, t)=\mathbf{p}+\sum_{\tau, \mathbf{c}} \beta\left(\frac{t-t_{\tau}}{\Delta_{\tau}}\right) B\left(\frac{\mathbf{p}-\mathbf{p}_{\mathbf{c}}}{\boldsymbol{\Delta}_{\mathbf{c}}}\right) \boldsymbol{\omega}_{\tau, \mathbf{c}}
$$

138 where $\boldsymbol{\omega}$ is an array of the control grid coefficients, acting as parameters of the B-spline, ${ }_{139} \mathbf{c}$ the spatial index and $\tau$ the temporal index, $\left(\boldsymbol{\Delta}_{\mathbf{c}}, \Delta_{\tau}\right)$ the width of the functions in each 140 dimension. This transformation model ensures both temporal and spatial consistency and 141 smoothness without compromising the local motion recovery due to its local control property. 142 More importantly, an estimate of small displacement or deformation can be achieved through ${ }_{143}$ the underlying interpolation between the control points. Note that to keep the continuity at 144 both ends of the cycle $\left(t_{\tau_{\min }}=0\right.$ and $\left.t_{\tau_{\max }}=1\right)$, we need to impose a pseudo-cyclic condition ${ }_{145} \boldsymbol{\omega}_{\tau_{\min }, \mathbf{c}}=\boldsymbol{\omega}_{\tau_{\max }, \mathbf{c}}$. A simple implementation is to extend the range of the transformation 146 model on the temporal axis at both ends, as illustrated in Fig. 1(a).

${ }_{147}$ For each $I_{t_{k}}$, a corresponding digitally reconstructed radiograph (DRR), $\tilde{I}_{t_{k}}$, is calculated 148 to simulate the X-ray angiography through a ray casting process ${ }^{23}$. For the rotational angiog149 raphy (RA) sequence, their projection geometry is known for each projection, including the 150 X-ray source position, the projection detector position, and the rotational orientation. We 
151 denote by $\tilde{\mathcal{I}}$ the entire DRR sequence, which is iteratively modified to match the measured 152 projection sequence $\mathcal{I}$ for an optimal estimation of $\hat{\boldsymbol{\omega}}$ :

$$
\hat{\boldsymbol{\omega}}=\underset{\boldsymbol{\omega}}{\operatorname{argmin}}\{M(\boldsymbol{\omega}, \mathcal{I}, \tilde{\mathcal{I}})\}
$$

153 where $M$ is the similarity metric between two mapping regions. Mutual information ${ }^{24,25}$ 154 was used as the metric. Since the registration matches simultaneously all the projections, 155 sampled points from the entire sequence are considered as within one region, forming a 156 single histogram. Therefore, instead of having one independent metric for each projection 157 pair, $M$ describes the similarity between the two sequences $\mathcal{I}$ and $\tilde{\mathcal{I}}$. Histograms are ap158 proximated using Parzen windows for the probability calculation ${ }^{26}$. The use of one metric 159 measuring the similarity between projection sequences makes the registration more robust 160 against local intensity variations (e.g noise and inhomogeneous contrast mixing) than con161 sidering similarities between individual projections separately. Note that due to the higher 162 spatial resolution in measured projections compared to the volumetric image, performing 163 the motion estimation from the projection space improves spatial accuracy of the recovered 164 motion field. In our case, a displacement equivalent to one pixel translates into approxi165 mately 0.3 voxel. The L-BFGS-B algorithm ${ }^{27}$ is used as the optimizer, due to its ability in 166 handling a very large number of parameters.

\section{${ }_{167}$ II.B. An efficient implementation}

Dealing simultaneously with such high-resolution 4D image, 2D measured projections 169 and DRRs, requires excessive memory and long computation time. For the method to be 170 practically applicable, reducing both of them without degrading the performance is desirable. ${ }_{171}$ Two strategies are jointly used in order to process the data of interest at each iteration during 172 the registration process. The fact that both computation and memory costs scale with the 173 amount of processed data makes these strategies efficient. They are further implemented on ${ }_{174}$ GPU so as to facilitate the clinical use of our technique at a reasonable execution time. The 175 main idea of the GPU implementation method is summarized in Appendix and a detailed 176 description can be found in ${ }^{20}$. 


\section{II.B.1. DRRs precomputation}

178 In 3DRA images, the structure of interest (e.g. an aneurysm or a vessel segment) is in the 179 order of millimeters, occupying a reduced region in the image (see an example in Fig. 2(a)). ${ }_{180}$ Thus, during the motion estimation process, the transformation can be applied only to a 181 volume of interest (VOI) while the rest of the volume remains unmodified. Provided that 182 the actual motion present outside the VOI is smaller than or of the same magnitude as the 183 motion in the VOI, it will not affect significantly the estimated motion. The reason is that ${ }_{184}$ due to the use of a sparse $B$-spline control-points grid, any motion outside may only 185 influence one projection in a particular direction while the motion of each control point is 186 the result of several projections. However, in order to simulate realistic X-ray projections, 187 voxels of the entire volume must be integrated at each iteration to update the DRRs. In 188 order to avoid redundant computation, for each pixel $\mathbf{x}$, the corresponding ray is split into 189 two parts: inside and outside the VOI. The constant outside part is precomputed, and at 190 each iteration only the inside part is integrated and updated to the sum of both parts. An 191 illustration is shown in Fig. 2(a) for a VOI containing an aneurysm. The speedup factor 192 using such pre-computation is the ratio between the ray segment length crossing the entire 193 volume and that of the VOI. The memory reduction rate is also expected to scale with this 194 factor.

\section{${ }_{195}$ II.B.2. Object-adaptive region-of-interests}

196 A common approach to accelerate the metric computation is to subsample the images. ${ }_{197}$ Uniform subsampling is not the most efficient method, and special attention should be paid 198 to reduce the calculation of the metric and its derivatives by sampling, for example the 199 object of interest ${ }^{28}$ or its edges ${ }^{29}$. We follow this strategy by encouraging dense sampling of 200 image regions that strongly influence the metric. Since morphology changes of the aneurysm 201 or vessel wall are reflected on the contrast enhanced lumen boundaries, two object-adaptive 202 sampling regions are introduced: the projected object $\left(\mathrm{S}_{\mathrm{OR}}\right)$ and the projected boundary ${ }_{203}\left(\mathrm{~S}_{\mathrm{BR}}\right)$. Consequently, the typical projected VOI, denoted as $\mathrm{S}_{\mathrm{VR}}$, for the computation of the 204 metric are replaced by the sequences of pixels from the sampling regions $\mathrm{S}_{\mathrm{OR}}$ or $\mathrm{S}_{\mathrm{BR}}$. An 205 illustration of these regions is shown in Fig. 2. 
There are many techniques automatically delineate such regions. Note that the accurate 207 definition of the sampling regions in $2 \mathrm{D}$ is not crucial for our method, as our interest is to 208 quantify 3D morphological changes. And since a reference image is available in 3D, we first 209 obtain one approximated 3D shape of the region using a threshold-based method, and then 210 define the region by simply projecting it on each projection. A unique property of a 3DRA 211 volume is that, looking at the histogram of this 3D image, there is a sharp differentiation 212 of the contrast agent (CA) filled regions (i.e. aneurysms and vessels) from the background. ${ }_{213}$ This results in clearly separated classes with the CA filled regions mapped to high voxel 214 value range and the background to low voxel value range ${ }^{10,30}$. Meanwhile, on the contrast 215 filled boundaries in the projection images, in general a region of progressive intensity change 216 exists. This is mainly due to the changes in length of the X-ray traversing the contrast-filled ${ }_{217}$ region on the boundaries, resulting in a continuous change of the accumulated attenuation. ${ }_{218}$ Consequently, this results in a similar pattern in the 3D reconstructed volume. Based on this 219 observation, the $S_{\mathrm{OR}}$ is calculated for each projection as follows. First, a boundary value of 220 the studied object is selected by identifying the CA filled regions from the histogram. Second, 221 on the corresponding ray for a specific pixel, as long as there is one sampled point having 222 larger intensity than this boundary value, the pixel is considered to be part of the $\mathrm{S}_{\mathrm{OR}}$. The 223 obtained region is comparable to the projected "shadow" of a 3D object from thresholding. ${ }_{224}$ Similarly for the $\mathrm{S}_{\mathrm{BR}}$, we first obtain two of these regions from different threshold values, by 225 repeating the process of the $\mathrm{S}_{\mathrm{OR}}$ region for two thresholds. One overestimates (i.e. higher 226 threshold) and the other underestimates (i.e. lower thresholds) the contrast filling region. ${ }_{227}$ The $\mathrm{S}_{\mathrm{BR}}$ region is obtained by subtraction of the two resulting regions. These boundary 228 identifying values or thresholds in the histogram can be obtained empirically or using e.g. ${ }_{229}$ Otsu's method ${ }^{31}$. Note that this gradually changing intensity pattern on the boundaries 230 between the contrast-filled region and the background also helps the recovery of a subvoxel 231 displacement estimated through the deformation of the reference image. The reason is 232 that such an intensity function follows a smooth transition that gives information on the 233 boundaries at a finer scale than the voxel grid, i.e. subvoxel resolution. 


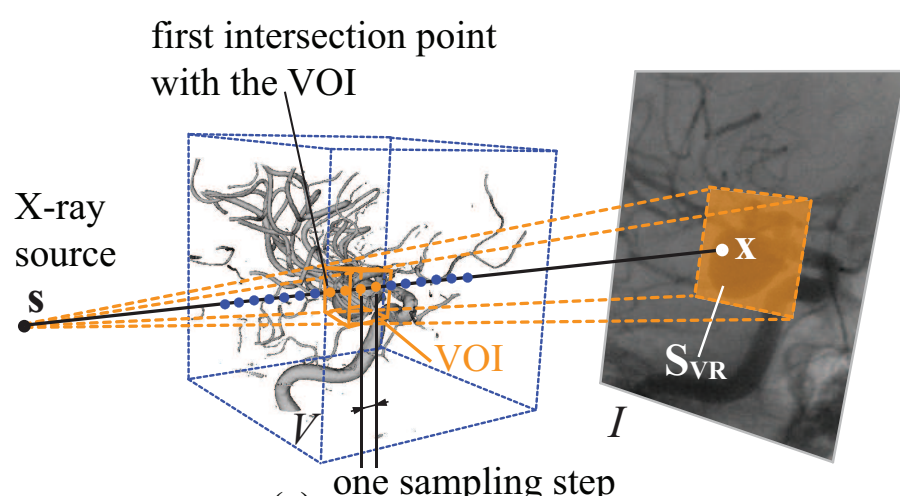

(a)

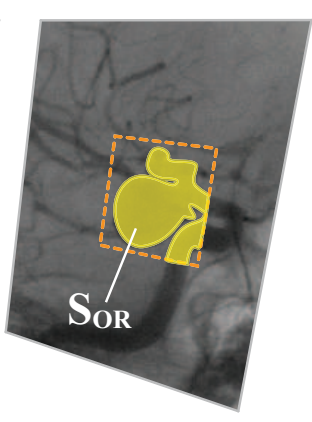

(b)

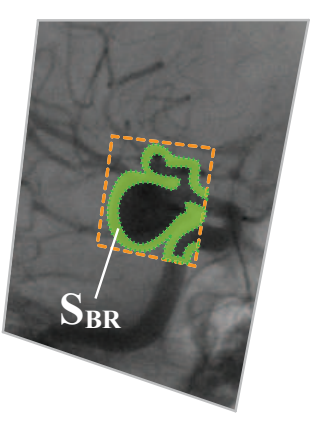

(c)

FIG. 2. (a) An illustration of the DRRs computation process. For each pixel $\mathbf{x}$, the corresponding ray is split into two parts: inside and outside the VOI. The constant outside part is precomputed, and at each iteration only the inside part is integrated and updated to the sum of both parts. The sampling region $\mathrm{S}_{\mathrm{VR}}$ contains the projected VOI. We introduce here two object-adaptive sampling regions: (b) the projected object region $\mathrm{S}_{\mathrm{OR}}$ and (c) the projected boundary region $\mathrm{S}_{\mathrm{BR}}$.

\section{${ }_{234}$ II.C. Strain map computation}

A number of mechanical and anatomical parameters can be used to characterize the 236 morphological and dynamic wall properties of the vasculature. We consider the strain map 237 extracted from the non-rigid wall motion estimation as a simplified but adequate way towards ${ }_{238}$ characterizing the vascular wall tissue. Such quantities provide a measure of the relative 239 deformation to which the arterial wall is exposed.

240 We study the distension of the vascular wall, which is related to the changes in wall 241 thickness. This relationship is more evident, for instance, under the volume-preserving 242 assumption as in $^{3}$, where the radial Cauchy strain is used. Specifically, it is computed 243 from triangular meshes that are extracted from the estimated volume images. Assuming 244 the volume of the material is preserved, the changes of the area $A_{t r}$ for each triangle are 245 inversely proportional to the changes in wall thickness $L_{w}: A_{t r} \times L_{w}=A_{t r}^{\prime} \times L_{w}^{\prime}$. Thus the ${ }_{246}$ radial Cauchy strain $\varepsilon_{c}$ is calculated as:

$$
\varepsilon_{c}=\frac{\Delta L_{w}}{L_{w}}=\frac{-\Delta A_{t r}}{A_{t r}^{\prime}}
$$

${ }_{247}$ where $\Delta L_{w}=L_{w}^{\prime}-L_{w}$ and $\Delta A_{t r}=A_{t r}^{\prime}-A_{t r}$. This means that the strain value is positive 248 if the material is stretched, or negative if it is compressed. 
249 III. VALIDATION

\section{III.A. Experimental data}

251 Our method has been currently applied to cerebrovascular wall motion with a particular 252 emphasis on cerebral aneurysm pulsation. We present here experiments on in silico and in 253 vitro aneurysm models, and also in vivo patient data.

${ }_{254}$ In silico: Twelve cases of digital aneurysm phantom models were created with dome di255 ameters of 8, 10, and $12 \mathrm{~mm}$ and parent vessel diameter of $4 \mathrm{~mm}$. They also have an emerging 256 bleb on the dome. The phantom motion was modeled as smooth geometry changes accord${ }_{257}$ ing to a sinusoidal pulsation waveform and was sampled at a finite number of time points. 258 According to the values on in vivo data presented in recent studies ${ }^{7-9}$, maximum pulsation 259 amplitudes were set to be $1 \%-4 \%$ of the dome diameter (i.e. $0.08-0.48 \mathrm{~mm}$ ). A sequence ${ }_{260}$ of volume images with an isotropic spacing of $0.3 \mathrm{~mm}$ was generated from the sequence of 261 ground-truth geometries. Voxel intensities were obtained as a function of the signed dis262 tance from the voxel to the object surface. The result is an image with a constant value 263 inside the object and another value outside, but with a blurred band of $0.5 \mathrm{~mm}$ around the 264 object boundary. Afterwards, this ground-truth volume sequence was used to generate the 265 synthetic measured projections with $0.16 \mathrm{~mm}$ spacing. In order to simulate other attenuated 266 vessels, air, bones, and soft tissues, we embedded the phantom images into a 3DRA patient 267 image that serves as background. An illustration is shown in Fig. 3(a). Once each phantom 268 was placed within the patient image, the voxels corresponding to aneurysm and vessel were 269 set to a typical intensity value of the CA filled regions.

270 In addition, in this paper we simulated spurious projection intensity variations in order 271 to analyze the sensitivity of our method and compare with other techniques. Such intensity 272 inhomogeneity is in general caused by the contrast filling following the blood flow. However, 273 the instantaneous local inhomogeneity might be caused by multiple factors. In order to 274 simulate realistic intensity variations, we sampled the RA image intensities from a patient 275 data where the aneurysm dome presented substantial nonuniform intensities including strong 276 blood turbulence. For the phantom dome region in each measured projection, an image patch 277 of the same shape was taken from the dome of the patient case and mapped directly to the 278 phantom image (Fig. 3(b)). 


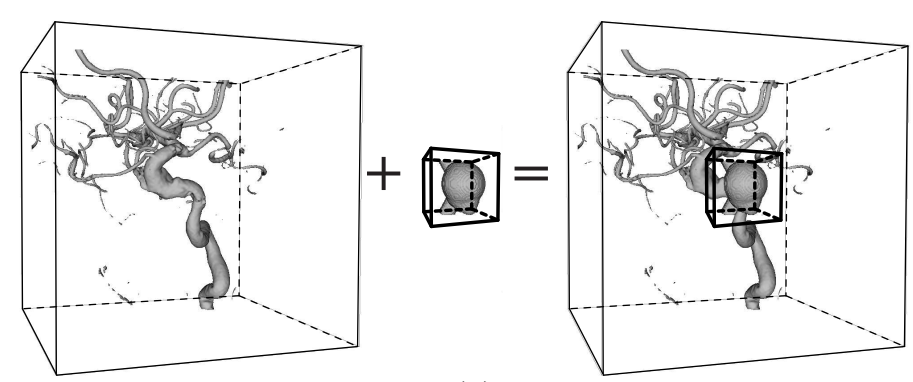

(a)

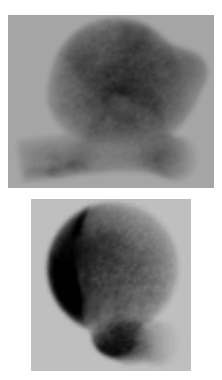

(b)

FIG. 3. (a) An example of an in silico phantom image, where the phantom model is embedded into a 3DRA patient image. (b) Projections with contrast inhomogeneity synthesized based on a RA patient data with strong blood turbulence.

In vitro: A silicone side-wall aneurysm phantom (Elastrat, Geneva, Switzerland) was 280 used. The model has a spherical dome with $10 \mathrm{~mm}$ diameter and a straight cylindrical 281 parent vessel with $4 \mathrm{~mm}$ diameter. It was placed in a rectangular container with dimensions 282 comparable to a human head. The container was water-filled to mimic the attenuation 283 of head tissue. In addition, two other phantoms with straight tubes were also placed in 284 the container to simulate background. The phantom was water-filled and connected to a ${ }_{285}$ customized pulsatile pump, a continuous flow pump (Elastrat, Geneva, Switzerland), and a 286 liquid tank to create a continuous and pulsatile flow circuit (Fig. 4(a)).

287 The image acquisitions were performed using an Allura Xper FD20 scanner (Philips ${ }_{288}$ Healthcare, Best, The Netherlands) equipped with a $220 \mathrm{~mm}$ detector field of view (diagonal 289 dimension) allowing a coverage of $75 \mathrm{~mm}$ of a cubic volume during a single rotation. For 290 these acquisitions, the injection protocol consisted of $18 \mathrm{~mL}$ of iodinated contrast material 291 (Iomeron 400, Bracco Imaging SpA, Milan, Italy) with a flow rate of 3mL/s. RA imaging 292 was performed at a frame rate of $30 \mathrm{~Hz}$ during contrast injection, with a $2 \mathrm{~s}$ delay. These 293 settings of the model and the imaging conditions give a realistic amount of scattering, 294 beam hardening and noise. An example RA image of the in vitro phantom is shown in ${ }_{295}$ Fig. 4(b). In total, 121 images were acquired $\left(1024^{2}\right.$ pixels with $(0.154 \mathrm{~mm})^{2} /$ pixel $)$ spanning ${ }_{296} \sim 210^{\circ}$ along the gantry trajectory, from which a $3 \mathrm{D}$ volume of $256^{3}$ voxels $\left((0.3 \mathrm{~mm})^{3}\right.$ 297 per voxel) was reconstructed. X-ray source and detector positions were recorded for each 298 projection, allowing the spatial relationship between the reconstructed reference volume and 299 each projection to be known. The scanning procedure and the imaging parameters of the 


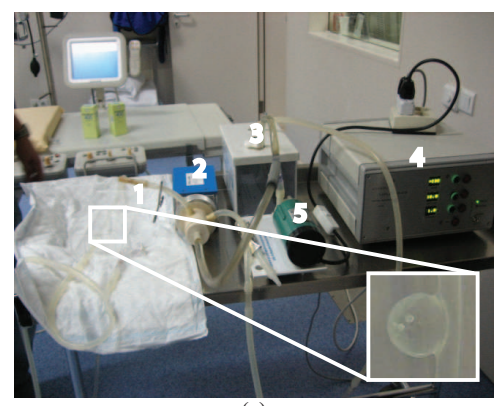

(a)



FIG. 4. (a) In vitro phantom experiments setup: 1. the silicone side-wall aneurysm; 2. the customized pulsatile pump; 3. the liquid tank; 4. the pulsatile signal generator; 5 . the continuous flow pump. (b) An example X-ray angiography of the in vitro phantom. The phantom was placed in a water-filled rectangular container (arrow head) with dimensions comparable to a human head. Two additional aneurysm phantoms with straight tubes (arrows) were also placed in the container, to act as background.

300 system followed a standard clinical protocol, which were also used for the in vivo cases 301 presented below. Detailed values are summarized in Table. I.

302 Three acquisitions were performed at different pump piston movement settings, resulting 303 in three phantom pulsation states: large pulsation (LP), small pulsation (SP), and non304 pulsation (NP). Although exact aneurysm pulsation amplitudes were unknown, the pulsation 305 range was in accordance with the expected range from visual inspection.

306 In vivo: Two 3DRA acquisitions from two patients with cerebral aneurysms were an307 alyzed in this paper. Both examinations were collected at Rothschild Foundation Paris, 308 using an Allura Xper FD20 scanner (Philips Healthcare, Best, The Netherlands). For these 309 examinations, the injection protocol consisted of $24 \mathrm{~mL}$ of contrast agent (Iomeron 350, 310 Bracco Imaging SpA, Milan, Italy) with a flow rate of $4 \mathrm{~mL} / \mathrm{s}$, with a $2 \mathrm{~s}$ delay. Patients 311 were under general anesthesia during the whole examination. We have estimated motion at 312 various locations as indicated in Fig. 5. Three types of motion were visually observed from 313 these regions: aneurysm wall motion, vessel wall motion, and catheter tip displacement. 314 For patient \#1, aneurysm motion could not be confirmed from the RA sequence, but we 315 observed it from an available digital subtraction angiography (DSA) sequence. For patient 316 \#2, aneurysm motion was not visible in the RA sequence, but we did observe vessel motion 317 and longitudinal displacements of the catheter. 
TABLE I. 3DRA imaging settings for the in vitro and in vivo data, using the Allura FD20 imaging system.

\begin{tabular}{|c|c|c|}
\hline Parameters & Unit & Value \\
\hline Tube Voltage & $\mathrm{KV}$ & $78-89$ \\
\hline Tube Current & $\mathrm{mA}$ & $180-280$ \\
\hline Exposure Time & $\mathrm{ms}$ & $6-8$ \\
\hline Detector Dose & nGy/fr & $\sim 200$ \\
\hline Detector Format & $\mathrm{cm}$ & 22,27 \\
\hline Focal Spot Size & $\mathrm{mm}$ & 0.4 \\
\hline Source-To-Isocenter Distance & $\mathrm{mm}$ & $\sim 810$ \\
\hline Source-To-Detector Distance & $\mathrm{mm}$ & $\sim 1195$ \\
\hline Geometric Magnification & - & $\sim 1.475$ \\
\hline Rotation Range & $\circ$ & $\sim 210$ \\
\hline Number of Projections & - & 121 \\
\hline Frame Rate & fps & 30 \\
\hline Pixel Spacing & $\mathrm{mm}$ & 0.154 \\
\hline Voxel Spacing & $\mathrm{mm}$ & 0.3 \\
\hline Contrast Injection Time & $\mathrm{s}$ & 6 \\
\hline Contrast Injection Rate & $\mathrm{mL} / \mathrm{s}$ & $3-4$ \\
\hline Iodine Density & $\mathrm{mg} / \mathrm{mL}$ & $350-400$ \\
\hline Collimator Filter (Alu) & $\mathrm{mm}$ & 1.0 \\
\hline Collimator Filter $(\mathrm{Cu})$ & $\mathrm{mm}$ & 0.1 \\
\hline Anti-Scatter Grid & $\mathrm{lp} / \mathrm{cm}$ & 80 \\
\hline
\end{tabular}

318 For all the experiments tested on these data, we chose a VOI of approximately $50^{3}$ voxels. 319 The number of sampled pixels in the sampling regions $\mathrm{S}_{\mathrm{VR}}, \mathrm{S}_{\mathrm{OR}}$ and $\mathrm{S}_{\mathrm{BR}}$ at each projection 320 view were in the order of 5000, 3000 and 500, respectively. The $B$-spline control point grid 321 spacing was about $1.5 \mathrm{~mm}$ for the spatial dimension, and $10-12.5 \%$ of the canonical motion 322 cycle for the temporal dimension. 

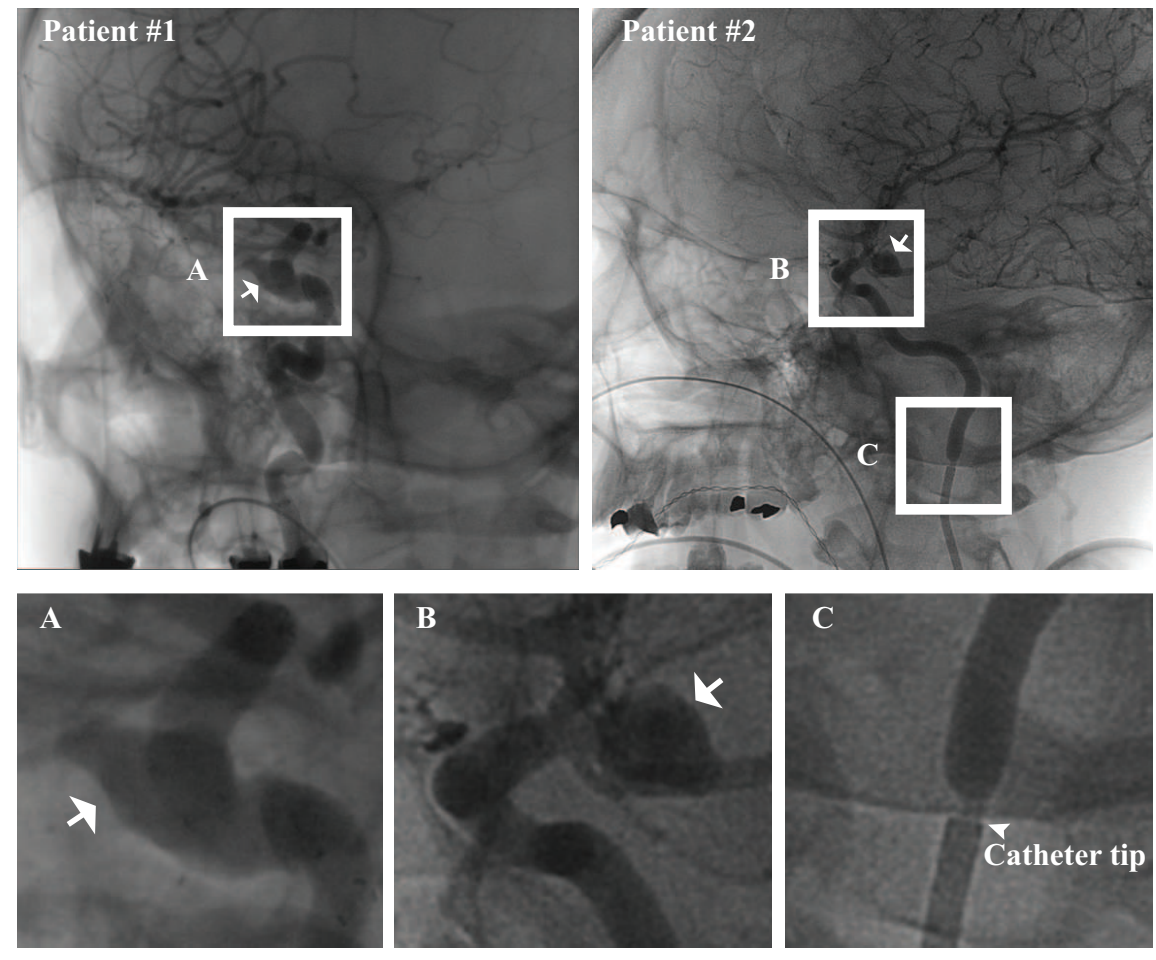

FIG. 5. Details of in vivo datasets, indicating with arrows the aneurysms and with an arrow head the catheter tip. Data from two patients are used in this work, where our method has been applied to different regions: (A) aneurysm with visible motion; (B) aneurysm without visible motion; (C) vessel segment with visible motion, and the imaged catheter (lower part) with longitudinal displacement.

\section{${ }_{323}$ III.B. Accuracy evaluation}

324 In order to quantitatively evaluate the accuracy of the estimated motion, a set of deformed $3253 \mathrm{D}$ volume images at discrete time points was extracted according to the estimated 4D 326 transformation. A relative error was measured at each time point $t$ as a percentage of the 327 pulsation range,

$$
e(t)=\left(m_{r}(t)-m_{g}(t)\right) / \hat{m}_{g} \times 100 \%
$$

328 where $m_{g}(t)$ is the ground-truth pulsation measurement (e.g., volume changes) at $t, m_{r}(t)$ 329 the corresponding estimated measurement, and $\hat{m}_{g}$ the variation range of $m_{g}(t)$ over the 330 canonical cycle.

331 In terms of volume change measurements, they were calculated using a method similar 




FIG. 6. Boxplots of $e_{V}$ at 16 equally distributed time points for 12 in silico phantom cases of different diameter $(8,10$, and $12 \mathrm{~mm})$ and maximum pulsation range (1\%-4\%).

332 to the one as $\mathrm{in}^{32}$, by transforming a binary mask image using the deformation field and $3 з 3$ subsequently summing up the intensities. The partial volume of the boundary voxels was ${ }_{334}$ calculated by dividing the sum of the interpolated intensities by the interval length.

\section{IV. RESULTS}

\section{${ }_{336}$ IV.A. In silico aneurysm wall motion}

337 For each case, we extracted 16 volume images at equally distributed time points along 338 the canonical motion cycle. As the ground-truth is known for these phantom data, a quan$3 з 9$ titative accuracy evaluation is possible. In the presented experiments, we used the relative 340 error in volume changes, $e_{V}$, calculated according to Eq. 4. Except for two cases in which 341 the maximum pulsation was below $0.1 \mathrm{~mm}$ (being the $8 \mathrm{~mm}$ and the $10 \mathrm{~mm}$ dome with $1 \%$ 342 maximum pulsation), the relative error in volume changes, $e_{V}$, was below 10\%, as can be 343 seen in Fig. 6.

344 In the example shown in Fig. 7, $e_{V}$ and the computational time are plotted for the 345 same number of registration iterations. In this experiment, we investigated the effects of 



FIG. 7. Performance evaluation on the use of a combination of three different schemes in terms of estimation error $e_{V}$ and computational time. The three schemes are: sampling regions $\left(\mathrm{S}_{\mathrm{VR}}, \mathrm{S}_{\mathrm{OR}}\right.$, $\mathrm{S}_{\mathrm{BR}}$ ), angular resolutions along the C-arm gantry trajectory (downsampling factor being 1-4), and the GPU implementation. Results were obtained from an in silico phantom with $12 \mathrm{~mm}$ diameter and $3 \%$ pulsation (i.e. maximum amplitude of $0.36 \mathrm{~mm}$ ).

346 using a combination of three different schemes: sampling region, angular resolution along 347 the C-arm gantry trajectory, and the GPU implementation. The angular resolution of the 348 measured projection sequence was downsampled by a factor of 1 to 4 . Results show similar 349 accuracy $\left(e_{V}<5 \%\right.$ up to three quartiles) achieved from the three sampling regions combined 350 with an angular resolution downsampling factor up to 3. Therefore, given the fact that less 351 projections can be used, it can be speculated that this also enables discarding the use of a few 352 undesirable projections, e.g. the ones with severe artifacts. No significant differences were 353 obtained when DRRs were generated using either the CPU or GPU implementation. The 354 slight GPU/CPU discrepancies can be attributed to the difference in data type specifications 355 between the processors ${ }^{33}$. Due to the speedup introduced at the GPU-based DRR generation 356 stage, the image registration process can be reduced by an additional factor of up to $2 \mathrm{x}$ with 357 respect to the corresponding CPU-based implementation. Therefore, the estimation results 358 for the complete motion cycle can be obtained in 5-10 minutes when using $\mathrm{S}_{\mathrm{BR}}$ on the GPU 359 DRR implementation using a downsampling factor of 3.

360 Fig. 8 shows the color maps of the amplitude wall displacements and the radial Cauchy 361 strain estimated at the maximum and minimum pulsation states of an in silico phantom 

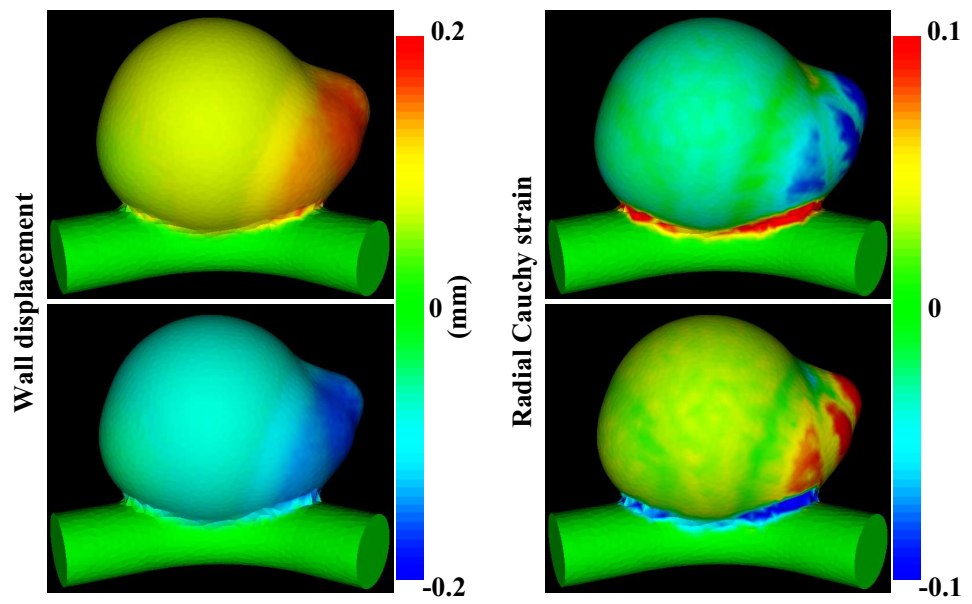

FIG. 8. Wall displacement amplitude and radial Cauchy strain at the maximum (top) and minimum (bottom) deformation states for an in silico phantom with diameter of $12 \mathrm{~mm}$ and pulsation of $3 \%$ (i.e. maximum amplitude of $0.36 \mathrm{~mm}$ ).

362 with diameter of $12 \mathrm{~mm}$ and pulsation of $3 \%$ (i.e. maximum amplitude of $0.36 \mathrm{~mm}$ ). In 363 regions with similar surface curvatures like the dome, the strain field presents a similar ${ }_{364}$ pattern to the displacement field, whereas in regions with higher curvatures, such as the 365 bleb and the neck, the strain scales faster. This suggests that the strain field might enhance 366 more efficiently regions having a different deformation pattern as strain is less insensitive to 367 passive motion but focuses on differential motion.

\section{${ }_{368}$ IV.B. In vitro aneurysm wall motion}

369 For the three pulsation states under evaluation, we obtained larger motion in the LP 370 case, smaller motion but with a similar pattern in the case of SP, and no motion for the NP 371 case. We show here the results of the LP case in Fig. 9. As the ground-truth is unknown, 372 the results are qualitatively presented. In Fig. 9(a-b), a measured projection is compared 373 with its corresponding DRRs calculated from the reference volume and from our estimated 374 volume. From the visual inspection in the projection space, our technique demonstrates its 375 ability in correcting the misalignment between the measured projection and the DRR. In 376 Fig. 9(c-d), color maps show the wall displacement amplitude and the strain at the time 377 point with the largest motion. An inhomogeneous wall displacement distribution is observed 378 and is especially concentrated on a lateral side of the aneurysm dome. This is caused by a 




(a)

- Measured $\square$ DRR from estimation



(b)
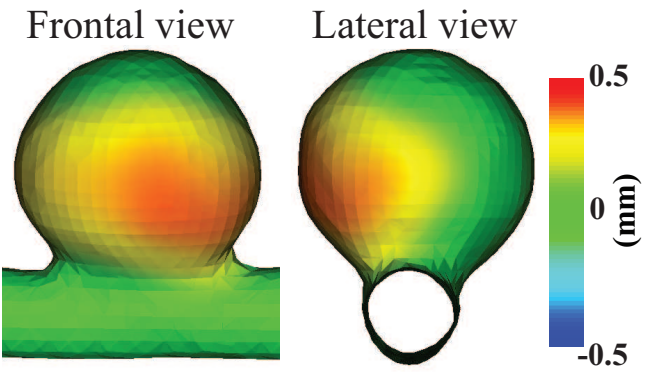

(c)

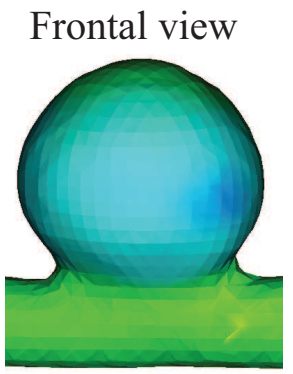

Lateral view

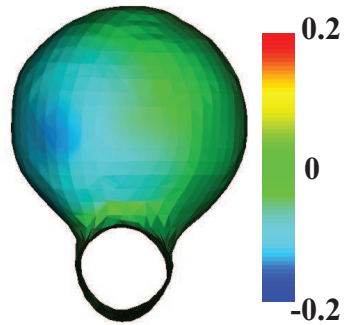

(d)

FIG. 9. Results from in vitro LP phantom: $(\mathrm{a}, \mathrm{b})$ Example of checkerboard images for the in vitro phantom comparing respectively the measured projection with the equivalent DRR computed from the reference volume and our estimation. The color maps of the wall displacement amplitude (c) and the radial Cauchy strain (d) for the frontal and lateral views at the instant presenting the largest displacement.

379 slight axial tilting of the phantom tube position during the acquisition. This is in agreement 380 with the reduced effect in terms of strain distribution observed at the same location, since 381 part of the displacements came from a global movement.

\section{${ }_{382}$ IV.C. In vivo cerebrovascular motion}

383 Our estimation recovered the visually observed aneurysm motion from patient \#1 and 384 vessel motion from patient \#2. For patient \#2, aneurysm motion was neither observed nor 385 recovered. Fig. 10 and Fig. 11 summarize the recovered motion from patients \#1 and \#2, 386 respectively. The color maps show the displacements and the radial Cauchy strain at the 387 end-systolic (ES) phase, which coincided with the cardiac time of the measured projections 388 where maximum motion was visually observed. This phase represented also the time of the 389 maximum motion estimated from our technique, as can be seen in the displacement curves 
(a)

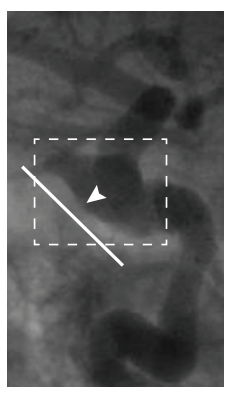

(b)



(c)

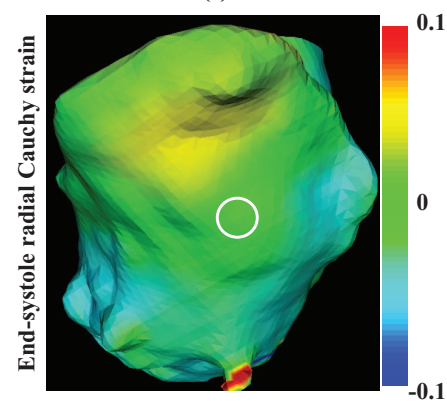



(d)

FIG. 10. Results of aneurysm wall motion in patient \#1. (a) A close view indicating the region where our motion estimation method has been applied (dashed frame) in an X-ray angiography. (b) The color map (line in (a) indicating the viewing plane) of the displacements around the endsystolic (ES) phase (indicated by the arrow in (d)). (c) The radial Cauchy strain at the same phase as in (b). (d) Aneurysm wall displacement amplitude over the cardiac cycle at the location indicated by the arrow head in (a) and the circles in (b,c).

390 over time in both figures. These curves show that the aneurysm in patient \#1 and the vessel 391 motion in patient \#2 presented a similar pattern with respect to the cardiac phases indicated 392 by the ECG signal. Spatially, for instance in Fig. 10, the motion was clearly observed in the 393 projections only in a small area of the aneurysm dome, which coincides with the maximum 394 estimated wall displacement region using our technique. Also, we observed that, in Fig. 11 395 the upper part of the vessel (i.e. internal carotid artery) did not show any visible motion. 396 This is consistent with the fact that this particular vessel segment, i.e. the petrous segment, 397 is surrounded by stiff bony structures preventing any motion at this location.

\section{${ }_{398}$ IV.D. Catheter displacement}

399 From the measured projections for patient \#2, we observed substantial longitudinal dis400 placement of the catheter, corresponding to the catheter tip moving vertically along the 401 direction of the vessel and following the blood flow. To further verify the feasibility of 402 our method in recovering general motion other than vascular wall motion from a rotational 

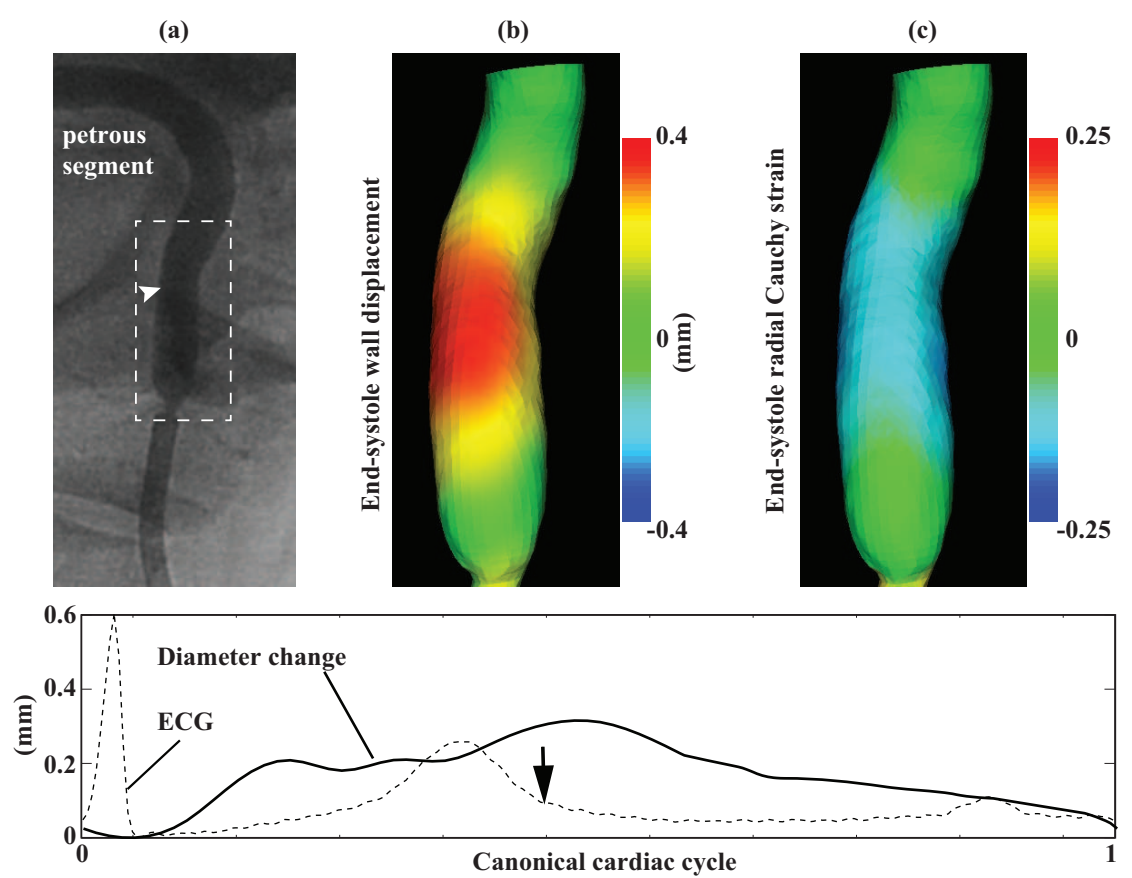

(d)

FIG. 11. Results of vessel wall motion in patient \#2. (a) A close view indicating the region where our motion estimation method has been applied (dashed frame) in an X-ray angiography. (b) The color map of the displacements around the end-systolic (ES) phase (indicated by the arrow in (d)). (c) The radial Cauchy strain at the same phase as in (b). (d) The vessel diameter change over the cardiac cycle at the location as indicated by the arrow head in (a).

403 angiography acquisition, we have applied it to the imaged catheter region and recovered 404 the displacement of the catheter tip. Results are shown in Fig. 12. The color maps show 405 respectively the displacements (Fig. 12(a)) at 10 equally sampled time instants over the 406 cardiac cycle. And the catheter tip displacement (along the vessel longitudinal direction) 407 is plotted with the ECG signal in Fig. 12(c). The cardiac phase when the maximum value 408 of this movement occurred was similar to the maximum vessel motion phase (Fig. 11(d)). 409 This confirms that the catheter moved back and forth according to the pulse of the blood ${ }_{410}$ flow. We have also plotted the calculated strain maps at the catheter surface in Fig. 12(b). ${ }_{411}$ As the strain represents deformation instead of rigid movement, it should be ideally zero ${ }_{412}$ everywhere and for all time instants. As expected, at the lower and homogeneous part of the 413 catheter, zero radial displacements and strain values were obtained. However, they were not 414 zero everywhere at the catheter tip. A first explanation for such behavior of the results is 


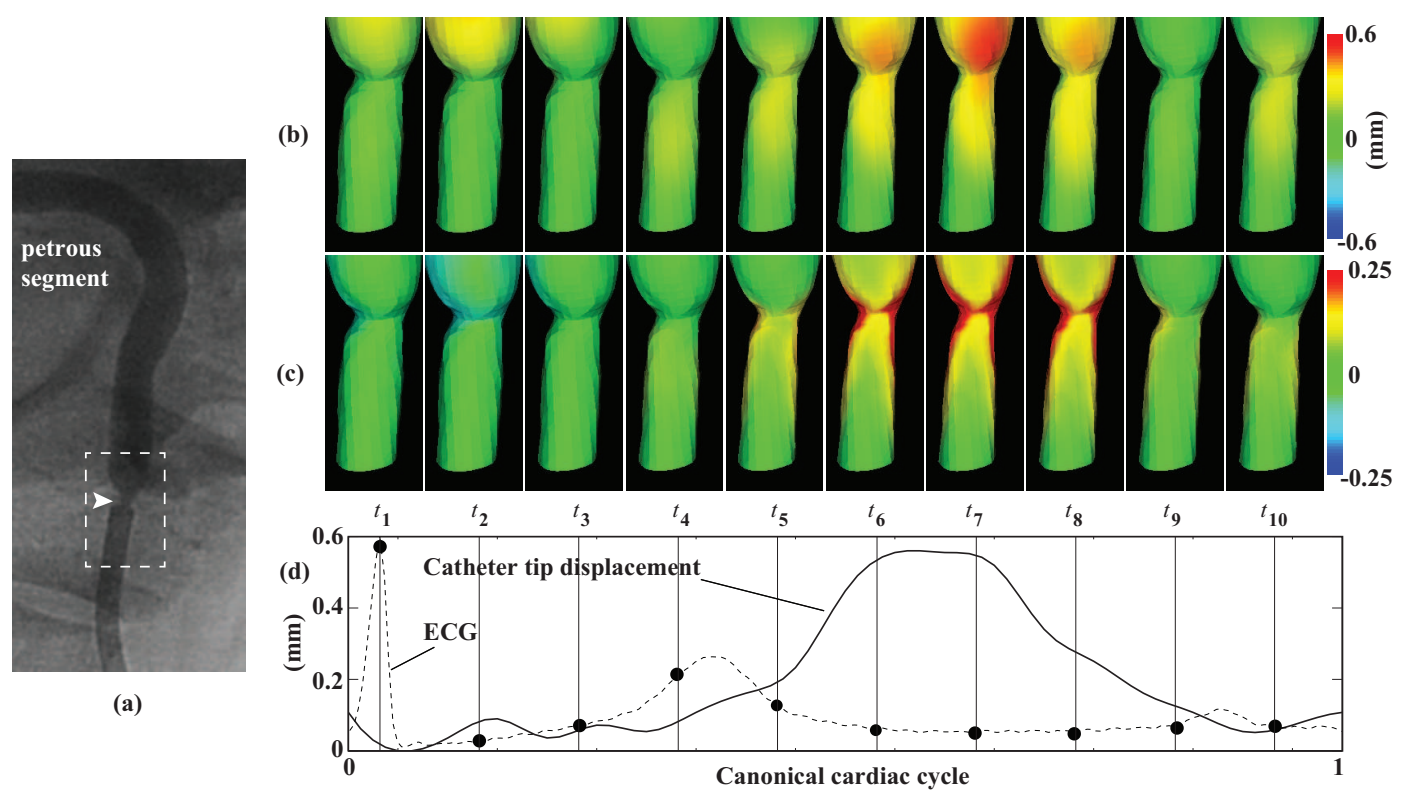

FIG. 12. Results of catheter tip movements in patient \#2. (a) A close view indicating the region where our motion estimation method has been applied (dashed frame) in an X-ray angiography. The color maps of the estimated catheter movements (b) and the strain (c) at ten selected time points. (d) The catheter tip (arrow head in (a)) longitudinal displacement plotted together with the ECG signal.

415 that the catheter used during the intervention had a flexible tip and therefore was prone to 416 deformation. Second, the estimated vessel motion was "propagated" to its immediate vicin${ }_{417}$ ity, the catheter tip, since the $B$-spline transformation provides a spatially smooth estimate 418 of the displacement field. And third, at the catheter tip, larger inhomogeneity of the con${ }_{419}$ trast agent mixing are expected, which in turn might affect our intensity-based registration 420 method.

\section{${ }_{421}$ V. DISCUSSION}

422 In silico pulsatile aneurysm phantom results have demonstrated that the estimation error 423 was below $10 \%$ in recovering motion in the sub-millimeter range, e.g. in the order of a voxel, 424 even from images with substantial intensity inhomogeneity. In vitro aneurysm phantom 425 experiments have allowed verifying that our method is able to detect whether an aneurysm ${ }_{426}$ pulsates or not. However, in a clinical environment, due to the lack of ground-truth motion 
${ }_{427}$ information, we were not able to validate quantitatively the performance of the method. ${ }_{428}$ Nonetheless and for the first time, experiments carried out on in vivo patient data pre${ }_{429}$ senting visible aneurysm and vascular wall motion as well as catheter tip movement, have 430 demonstrated the feasibility of our method for motion detection and recovery from RA. In ${ }_{431}$ regions where motion or deformation is impossible from an anatomical point of view, such 432 as the petrous segment and the catheter, the results were consistent with the expected zero 433 motion. In summary, although ground-truth was unknown for the in vitro and the in vivo 434 data, our results were qualitatively accurate. Given the realistic modeling of spatial and 435 temporal imaging conditions as well as the morphology and motion range, the performance 436 of our method on in silico data can be expected, to a certain extent, to be translatable ${ }_{437}$ to patient data acquired in a clinical situation.

${ }_{438}$ To facilitate the translation of this technique into clinical practice, we proposed the joint 439 use of two acceleration strategies together with their implementation on graphics processing 440 units. This has demonstrated a successful memory management and speedup for processing ${ }_{441}$ large 3D and 2D datasets from 3DRA acquisitions. These improvements allowed completing ${ }_{442}$ the motion estimation process for one entire cycle in 5-10 minutes without degrading the ${ }_{443}$ overall performance. More specifically, we obtained a 3-4x speedup from the precomputation ${ }_{444}$ of surrounding vascular structures outside the VOI, and a 10x from the use of $\mathrm{S}_{\mathrm{BR}}$. With 445 respect to the $\mathrm{CPU}$ implementation, an additional speed improvement of up to $2 \mathrm{x}$ was ${ }_{446}$ achieved by integrating the GPU generated DRRs in the motion estimation framework.

${ }_{447}$ Since the object-adaptive ROIs are calculated based on two selected voxel values as ${ }_{448}$ described in Section II.B.2, the potential influence of these intensity values on the estimation 449 is discussed here. Experiments were performed on an in silico phantom (dome diameter of ${ }_{450} 12 \mathrm{~mm}$ and maximum pulsation of $3 \%$ ) embedded in a 3DRA patient image. Voxel intensities ${ }_{451}$ of the phantom dome were set to be constant inside (i.e. a value belonging to the CA filled 452 region), and to be smoothly changing on the boundary, depending on the distance from the ${ }_{453}$ voxel to the ideal wall surface. Results are demonstrated on four $\mathrm{S}_{\mathrm{BR}}$ regions (denoted as ${ }_{454}$ R1-R4), chosen from different combinations of four sub-ranges equally spanning the intensity 455 range of the phantom. The lower boundary intensity value of R1 was chosen to be higher 456 than the actual boundary and thus was inside the phantom. That means, in R1 the aneurysm ${ }_{457}$ wall was not included, while in R2 to R4 the actual aneurysm wall was always included but 458 with the inner boundary identified by three different values spanning the aneurysm intensity 



FIG. 13. Comparison of the accuracy using four different $S_{B R}$ regions (denoted as R1-R4), chosen from different combinations of four sub-ranges equally spanning the intensity range of the phantom. Results in this figure were obtained from an in silico phantom embedded in a 3DRA patient image (see an illustration in Fig. 3(a)). The phantom has a diameter of $12 \mathrm{~mm}$ and the maximum pulsation of $3 \%$ (i.e. maximum amplitude of $0.36 \mathrm{~mm}$ ).

459 range. Detailed distributions of these four regions are illustrated on the histogram of the 460 reference volume image, as shown in Fig. 13. Their corresponding relative estimation error ${ }_{461} e_{V}$ values are also plotted in the figure. Results suggest that the choice of the voxel intensity 462 values for the boundary region does not affect much the estimation accuracy, when the 463 expected wall motion region is within the chosen $\mathrm{S}_{\mathrm{BR}}$. In the case of $\mathrm{R} 1$, larger errors were 464 obtained because this region excludes the intensity range of the aneurysm wall by focusing 465 on too high intensities.

${ }_{466}$ In the following, we discuss the performance comparison between a previous technique ${ }^{18}$ ${ }_{467}$ for 3D independent motion estimation at specific time points (denoted as ALG1) and our ${ }_{468}$ proposed 4D motion cycle estimation technique (denoted as ALG2). In general, similar 469 accuracy values could be expected using both techniques, since the plot shown in Fig. 7 470 presented comparable error values as reported in ${ }^{18}$. In terms of computational efficiency, 471 the time spent for a full $4 \mathrm{D}$ motion estimation in this paper is comparable to what is 472 needed for computing only one 3D estimation at a specific time point using ALG1. In the 

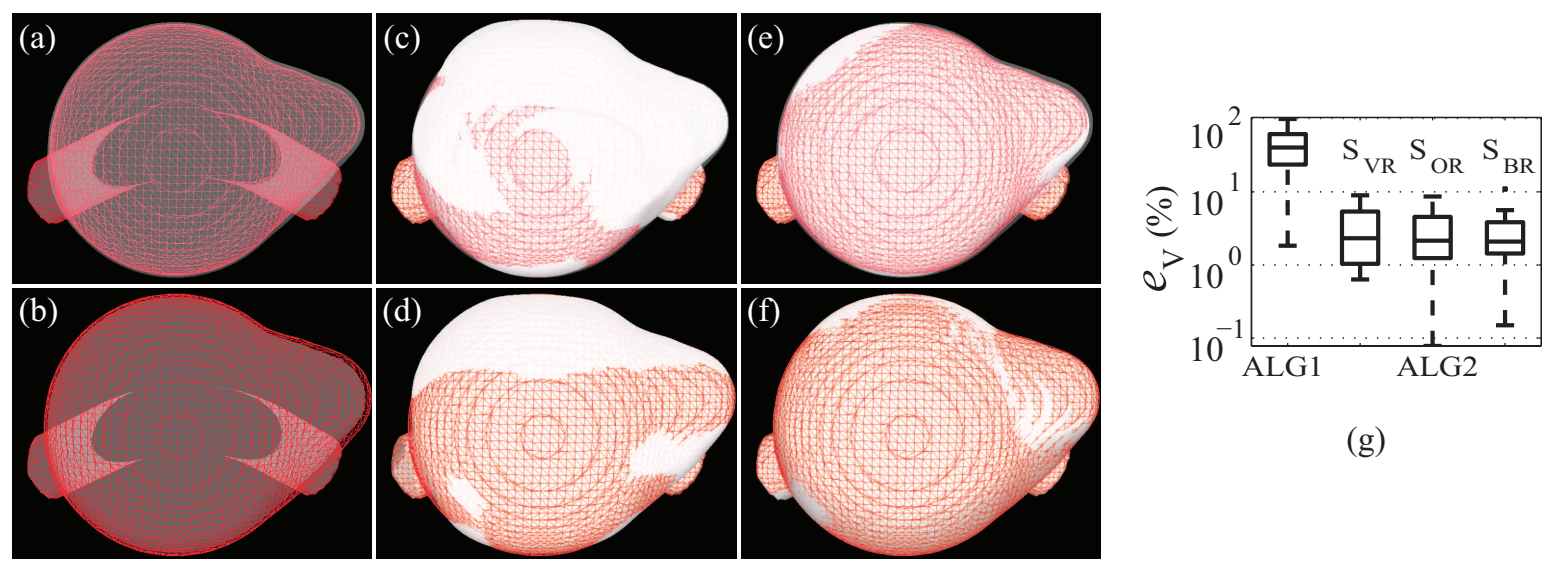

(g)

FIG. 14. Results comparing the influence of inhomogeneous contrast filling on the method in $^{18}$ (denoted as ALG1) and our present technique (denoted as ALG2), using an in silico phantom with diameter of $10 \mathrm{~mm}$ and pulsation of $4 \%$ (i.e. maximum amplitude of $0.4 \mathrm{~mm}$ ). Results at two instants are shown graphically: (a,c,e) minimum pulsation and (b,d,f) maximum pulsation. The ground-truth shape (wireframe representation) at each time instant is overlaid with: (a,b) the reference, $(\mathrm{c}, \mathrm{d})$ the estimation using ALG1, and $(\mathrm{e}, \mathrm{d})$ the estimation using ALG2. (g) Comparison of $e_{V}$ between ALG1 and ALG2 with the three sampling regions: $\mathrm{S}_{\mathrm{VR}}$, $\mathrm{S}_{\mathrm{OR}}$, and $\mathrm{S}_{\mathrm{BR}}$.

473 situation with large intensity variations in the contrast-enhanced regions in the projection 474 images, such as inhomogeneous contrast mixing, our method or ALG2 is however expected 475 to be more robust than ALG1. Results shown in Fig. 14 were obtained from the simulated 476 inhomogeneous contrast-filled images, as described in Section III.A. The relative volume ${ }_{477}$ error $e_{V}$ was below $10 \%$ using ALG2, whereas using ALG1 it was on average $50 \%$ or even 478 larger. This large difference is due to the fact that ALG1 failed to recover the motion from 479 such input images. This can be visually observed in Fig. 14 from the surface of the ground480 truth shapes at two example time instants overlaid with the estimations (i.e. maximum and ${ }_{481}$ minimum shape extension). Constrast inhomogeneity in this case induces an overestimation ${ }_{482}$ of the phantom motion using ALG1 in comparison with ALG2. This suggests that our ${ }_{483} 4 \mathrm{D}$ estimation is more robust to large image intensity inhomogeneity, both temporally and 484 spatially. Additionally, a slightly higher accuracy was obtained using the projected boundary ${ }_{485}$ region $\mathrm{S}_{\mathrm{BR}}$. This could be possibly due to the exclusion of inner regions with inhomogeneous 486 intensities, reducing the noise influence to the registration.

${ }_{487}^{48}$ As the expected cerebral aneurysm wall motion range is very small, the impact of other 
488 possible physiological motion that might affect the motion estimation needs to be discussed. ${ }_{489}$ The most intuitive one is respiratory motion, however in our application its impact is neg490 ligible. First, from the clinical examination protocol point of view, the respiratory induced 491 motion in the head is not likely to happen, given that the patient lies still, either under 492 general anesthesia or when instructed to hold their breath for a few seconds during ${ }_{493}$ the 3DRA acquisition (in our case $4 \mathrm{~s}$ ), with the head in an immobilizing headrest. Second, 494 from our methodology point of view, we use projections from one canonical cardiac cycle 495 that are built from multiple cardiac cycles, and we model this cycle by a $4 \mathrm{D}$ smooth and 496 continuous transformation. The method assumes pseudo-periodicity in such a way that acts 497 as a filter forcing the reconstructed motion to be just one canonical cycle. This, in effect, 498 helps to reinforce motion induced by blood pressure changes occurring over the cardiac 499 cycle, and meanwhile, produces an averaging of other physiological motion that does not 500 occur with the cardiac cycle, such as respiratory motion. In fact, it works in a similar way 501 as how standard 3DRA reconstructions ignore the existence of any kind of motion. This 502 reference reconstruction is reliable because the potential motion is small in comparison with 503 the size of the reconstructed objects. In our case, the estimated pseudo-periodic vascular 504 motion should be reliable while the spontaneous irregular non-periodic motion is small in 505 comparison with the periodic motion. These reasons can also justify the ignorance to the 506 possible irregular variation (or large deviations) of the cardiac cycles. Recently, after fol507 lowing over 30 cerebral aneurysm embolization interventions we have found an intrascan 508 heart beat variability below $1.5 \%$ on average and not exceeding $4 \%$. This variability is small 509 enough to be averaged or compensated by our method. Other movements throughout the 510 rotational run that might also have an influence is related to highly attenuated structures, 511 e.g. bones or the skull. In this case, the possibility and the amount of this motion varia512 tion are negligible, as the bone movement can be considered to be global and very small. ${ }_{513}$ Specifically because the skull is covering all the imaged region, and its material and mo514 tion can be assumed to be homogeneous. Furthermore, this effect is minimal under our 515 methodology framework, since the ray traverses through a highly contrast-enhanced object, 516 and the projection intensity is mostly determined by the accumulated attenuation of the 517 contrast-enhanced vessels. Therefore, the potential projection intensity variation caused by 518 the movement of bones for a specific projection pixel can be ignored in principle. This also 519 confirms that our acceleration strategy, the precomputation outside the region of interest 
520 is a reliable approximation. However, in the case that a substantial amount of any of ${ }_{521}$ the aforementioned motion occurs during the acquisition, the reliability of the estimated 522 vascular motion could be decreased.

523 In general for X-ray imaging applications, the variations of intrinsic detector performance ${ }_{524}$ parameters could probably play a role in the image quality, as has been studied intensively ${ }_{525} \mathrm{in}^{34-39}$. These parameters can provide characteristics that consider the complete imaging 526 system performance, including the effect of focal spot blurring, magnification and scatter. ${ }_{527}$ They have more pronounced effects for general applications with less image contrast ${ }^{34}$ or 528 small structures like stent struts (e.g. $0.1 \mathrm{~mm}$ or lower) ${ }^{35,37}$ using a microangiographic flu529 oroscopic imaging system ${ }^{37,38}$. In our case, the studied objects like selective CA enhanced 530 vascular structures are imaged with high dose and are highly contrasted. Also object size is 531 expected to be in a larger magnitude. Admittedly, the intrinsic spatial extent of the detector 532 limits the motion recovering of our technique to a certain range. But the use of a sparse set of ${ }_{533} B$-spline control points means that the estimated motion of each control point is determined 534 by many points along the object surface and boundary. This enables us to obtain a realistic 535 estimation of the wall motion whose magnitude is equivalent to small fractions of the total 536 system mean imaging aperture or unsharpness. Meanwhile, note that this limited resolution 537 of currently existing systems is expected to be improved in the future, which will enable 538 our method to estimate even smaller motion. This factor is reflected in the results shown in ${ }_{539}$ Fig. 6 on in silico phantom experiments. In this figure, at least for two phantom cases $(8 \mathrm{~mm}$ 540 and $10 \mathrm{~mm}$ with $1 \%$ motion for both), we were not able to recover correctly the motion. Fur541 ther resolution improvements and thus motion estimation with small magnitude could be ${ }_{542}$ expected when geometric unsharpness effects can be minimized either through reduction of 543 the focal spot size or reduction of the magnification. However, the options for a reduction 544 of the aforementioned two factors are limited. As this study serves to show the feasibility of ${ }_{545} 4 \mathrm{D}$ aneurysm wall motion estimation from rotational angiography, a more detailed analysis 546 of the impact of these factors on the estimation accuracy and robustness will be addressed 547 in future work and is beyond the scope of this paper.

548 The experimental results also emphasize the feasibility of performing strain analysis from 549 the estimated motion, making thus possible the use of this information for further estimating 550 elastic properties of the vascular wall, using for example an inverse problem approach ${ }^{3}$. ${ }_{551}$ Note that the strain map was not obtained through tracking individual points or tissue on 
552 the vascular wall. Thus, our approach for strain calculation through quantifying apparent 553 motion from images implies that the correspondences over time are approximations of the 554 same physical point.

\section{${ }_{555}$ VI. CONCLUSIONS}

556 This paper has presented a technique to recover $4 \mathrm{D}$ cerebrovascular wall motion that is ${ }_{557}$ in the order of sub-millimeter, from a single 3DRA acquisition within a clinically acceptable 558 computation time. Using this technique, the recovered motion is temporally and spatially 559 smooth, which also improves the robustness of the estimation to noise and intensity inhomo560 geneity. The subsequent strain calculation based on our motion estimation provides further ${ }_{561}$ progress towards the biomechanical modeling of the cerebrovascular wall. Our technique 562 also provides the possibility of detecting vascular wall abnormalities through direct visual563 ization of motion over time. It is highly desirable to have a technique that offers accurate 564 and robust in vivo estimates of such motion. In order to translate our method into a 565 clinical setting, future research efforts should be paid to validate our method on a larger 566 number of patient data sets.

\section{${ }_{567}$ ACKNOWLEDGMENTS}

568 The authors would like to thank Dr. Raphaël Blanc from Rothschild Foundation Paris, ${ }_{569}$ for the patient data acquisitions, Dr. D. Rüfenacht and L. Augsburger from Geneva Univer570 sity Hospitals, for providing the aneurysm silicone phantom and the pulsatile pump, J.-M. ${ }_{571}$ Dhieux and J.-P. Ruaud from Philips France for their technical support, and R. Hermans ${ }_{572}$ from Philips Healthcare (Best, The Netherlands) for 3DRA data software. They also thank ${ }_{573} \mathrm{R}$. Cárdenes for the helpful discussion. This work was partially supported by the CENIT${ }_{574}$ CDTEAM grant funded by the Spanish Ministry of Science and Innovation, partially gen575 erated in the framework of the @neurIST Integrated Project, which is co-financed by the

${ }_{576}$ European Commission (IST-027703), and partially supported by the Spanish Ministry of ${ }_{577}$ Science and Innovation (Ref. TIN2009-14536-C02-01), Plan E and FEDER. 


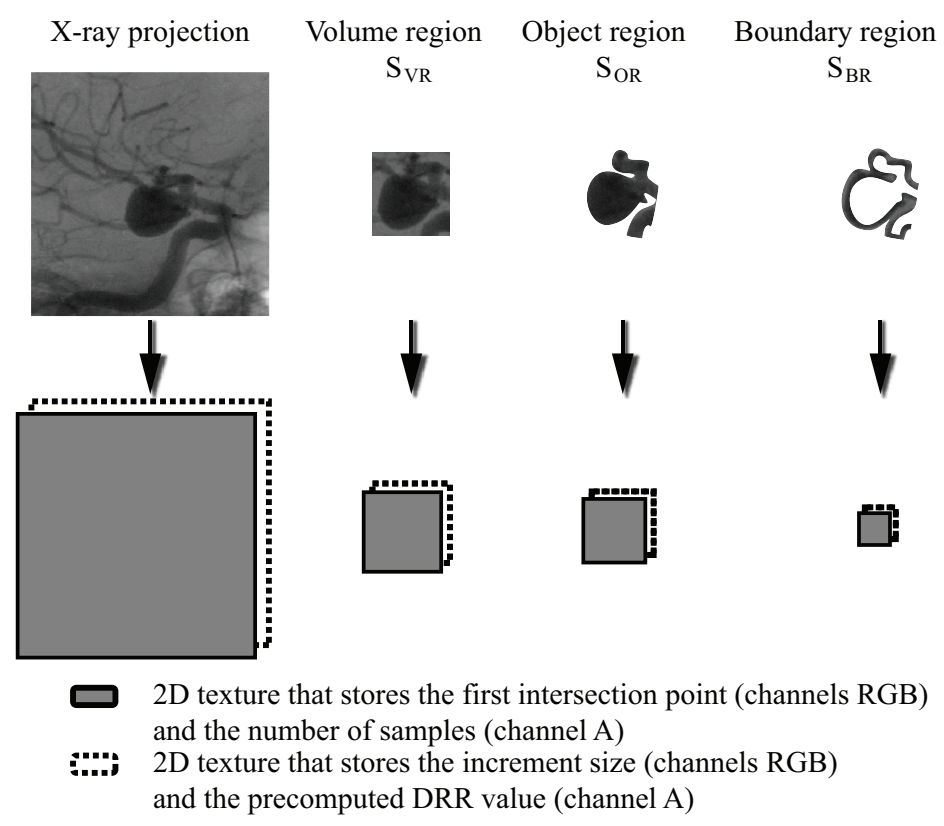

FIG. 15. An illustration of how information is repacked for the final DRRs computation for the proposed three structures of interest $\left(\mathrm{S}_{\mathrm{VR}}, \mathrm{S}_{\mathrm{OR}}\right.$ and $\left.\mathrm{S}_{\mathrm{BR}}\right)$ into $2 \mathrm{D}$ textures of decreasing sizes.

\section{${ }_{578}$ Appendix: GPU implementation of DRRs generation}

579 To further speedup the method, the DRRs generation combined with the acceleration 580 strategies is implemented on GPU and is integrated into the image registration process. ${ }_{581}$ We briefly describe the main idea of the method here. Unlike traditional GPU-based DRR ${ }_{582}$ generation methods ${ }^{40}$, our implementation also integrates the two previously introduced 583 strategies, thus benefits from both the GPU parallelization and the resultant memory re584 duction from these strategies.

${ }_{585}$ The method was implemented using the $\mathrm{Cg}$ (C for graphics) toolkit ${ }^{41}$ and on the pixel 586 shader units of a NVIDIA GeForce 8600 GT graphics card with 512MB of memory, hosted 587 by an Intel ${ }^{\circledR}$ Core $^{\mathrm{TM}} 2$ Quad CPU Q6600 2.40GHz with 4GB of memory. DRR pixel data 588 are stored as stream data in the format of textures, and fed to the GPU fragment units 589 so that each fragment works in parallel on a single pixel. Each texture element can store 590 up to four components, or the RGBA channels, as they are originally used to represent the ${ }_{591}$ red, green, blue, and alpha intensities of a color for rendering. In order to reduce redundant 592 calculations, we compute first a number of parameters that are constant when updating ${ }_{593}$ the DRRs during each iteration. As we equidistantly sample points on the ray (Fig. 2(a)), 594 only the first intersection point on the volume and the sampling step vector are needed, 
595 the remaining points can be derived in a straightforward manner. In total, eight constant ${ }_{596}$ parameters are needed for each pixel to calculate the DRRs: the first intersection point, ${ }_{597}$ the sampling step, the number of sampled points and the pre-computed DRR value. Since 598 we only calculate the pixel values within the ROIs $\left(\mathrm{S}_{\mathrm{VR}}, \mathrm{S}_{\mathrm{OR}}\right.$, or $\left.\mathrm{S}_{\mathrm{BR}}\right)$, these eight constant 599 parameters to calculate the pixel values in the ROI are re-packed into two $2 \mathrm{D}$ rectangular 600 textures of smaller sizes than the original projections (see an illustration in Fig. 15). They 601 are used in a GPU procedure that only performs the main loop over the VOI at every ${ }_{602}$ registration iteration. This way, the GPU fragment code remains short to maintain the ${ }_{603}$ stream processing advantage with respect to its equivalent CPU calculations.

\section{REFERENCES}

605

606

607

608

${ }^{1}$ D. M. Sforza, C. M. Putman, and J. R. Cebral, "Hemodynamics of cerebral aneurysms," Annu. Rev. Fluid. Mech. 41, 91-107 (2009).

${ }^{2}$ J. C. Lasheras, "The biomechanics of arterial aneurysms," Annu. Rev. Fluid. Mech. 39, 293-319 (2007).

${ }^{3}$ S. Balocco, O. Camara, E. Vivas, T. Sola, L. Guimaraens, H. A. F. Gratama van Adel, C. B. Majoie, J. M. Pozo, B. H. Bijnens, and A. F. Frangi, "Feasibility of estimating regional mechanical properties of cerebral aneurysms in vivo," Med. Phys. 37, 1689-1706 (2010).

${ }^{4}$ M. Kroon and G. A. Holzapfel, "Estimation of the distributions of anisotropic, elastic properties and wall stresses of saccular cerebral aneurysms by inverse analysis," Proc. R. Soc. A 464, 807-825 (2008).

${ }^{5}$ L. Dempere-Marco, E. Oubel, M. Castro, C. Putman, A. F. Frangi, and J. R. Cebral, "CFD analysis incorporating the influence of wall motion: application to intracranial aneurysms," in R. Larsen, M. Nielsen, and J. Sporring (Eds.): MICCAI2006, LNCS4191 (2006) pp. 438-445.

${ }^{6}$ F. Ishida, H. Ogawa, T. Simizu, T. Kojima, and W. Taki, "Visualizing the dynamics of cerebral aneurysms with four-dimensional computed tomographic angiography," Neurosurgery 57, 460-471 (2005).

${ }^{7}$ T. Krings, P. Willems, J. Barfett, M. Ellis, N. Hinojosa, J. Blobel, and S. Geibprasert, "Pulsatility of an intracavernous aneurysm demonstrated by dynamic 320-detector row 
636

${ }_{646}{ }^{14}$ L. A. Feldkamp, L. C. Davis, and J. W. Kress, "Practical cone beam algorithms," J. Opt. 647 Soc. Am. A 6, 612-619 (1984).

${ }^{648}{ }^{15}$ C. Rohkohl, G. Lauritsch, L. Biller, M. Prümmer, J. Boese, and J. Hornegger, "Inter649 ventional 4D motion estimation and reconstruction of cardiac vasculature without motion 650 periodicity assumption," Med. Image Anal. 14, 687-694 (2010).

${ }_{651}{ }^{16}$ R. Zeng, J. A. Fessler, and J. M. Balter, "Estimating 3-D respiratory motion from orbiting 652 views by tomographic image registration," IEEE Trans. Med. Imag. 26, 153-163 (2007).

${ }_{653}{ }^{17}$ J. Vandemeulebroucke, J. Kybic, P. Clarysse, and D. Sarrut, "Respiratory motion es${ }_{654}$ timation from cone-beam projections using a prior model," in G.-Z. Yang et al. (Eds.): ${ }_{655}$ MICCAI2009, LNCS5762 (2009) pp. 365-372.

${ }^{656}{ }^{18}$ C. Zhang, M.-C. Villa-Uriol, M. De Craene, J. M. Pozo, and A. F. Frangi, "Morphody- 
${ }_{665}{ }^{21}$ M. Unser, "Splines: a perfect fit for signal and image processing," IEEE Signal Process. 666 Mag. 16, 22-38 (1999).

${ }_{667}{ }^{22}$ D. Rueckert, L. I. Sonoda, C. Hayes, D. L. G. Hill, M. O. Leach, and D. J. Hawkes,

${ }_{670}{ }^{23}$ R. L. Siddon, "Fast calculation of the exact radiological path for a three-dimensional CT 671 array," Med. Phys. 12, 252-255 (1985).

${ }_{672}{ }^{24} \mathrm{P}$. Viola and W. M. Wells, "Alignment by maximization of mutual information," Int. J. 673 Comput. Vision 24, 137-154 (1997).

${ }^{674}{ }^{25}$ F. Maes, A. Collignon, D. Vandermeulen, G. Marchal, and P. Suetens, "Multimodality 675 image registration by maximization of mutual information," IEEE Trans. Med. Imag. 16, $676 \quad 187-198(1997)$.

${ }_{677}^{26}$ D. Mattes, D. R. Haynor, H. Vesselle, T. K. Lewellen, and W. Eubank, "PET-CT image 678 registration in the chest using free-form deformation," IEEE Trans. Med. Imag. 22, 120$679128(2003)$.

${ }^{680}{ }^{27}$ C. Zhu, R. H. Byrd, and J. Nocedal, "L-BFGS-B: Algorithm 778: L-BFGS-B, FORTRAN 681 routines for large scale bound constrained optimization," ACM Trans. Math. Software 23, $682 \quad 550-560(1997)$.

${ }_{683}^{28}$ M. R. Sabuncu and P. J. Ramadge, "Gradient based nonuniform subsampling for ${ }_{684}$ information-theoretic alignment methods," in Proc. Int. Conf. IEEE Eng. Med. Biol. Soc., 685 Vol. 3 (2004) pp. 1683-1686.

${ }_{686}{ }^{29}$ R. Bhagalia, J. A. Fessler, and B. Kim, "Accelerated nonrigid intensity-based image 687 registration using importance sampling," IEEE Trans. Med. Imag. 28, 1208-1216 (2009). ${ }^{688}{ }^{30}$ H. Bogunović, A. G. Radaelli, M. DeCraene, D. Delgado, and A. F. Frangi, "Image 
${ }^{691}{ }^{31}$ N. Otsu, "A threshold selection method from gray-level histograms," IEEE Trans. Syst. 692 Man. Cybern 9, 62-66 (1979).

${ }_{693}{ }^{32}$ M. Holden, J. A. Schnabel, and D. L. G. Hill, "Quantification of small cerebral ventricular

694

${ }_{696}^{33}$ IEEE, "IEEE standard for floating-point arithmetic," IEEE Std 754-2008 , 1-58 (2008).

${ }_{697}{ }^{34} \mathrm{~V}$. Rasche, B. Schreiber, C. Graeff, T. Istel, H. Schomberg, M. Grass, R. Koppe,

${ }^{709}{ }^{37}$ V. Patel, K. R. Hoffmann, C. N. Ionita, C. Keleshis, D. R. Bednarek, and S. Rudin, 710 "Rotational micro-CT using a clinical C-arm angiography gantry." Med. Phys. 35, 4757$7114764(2008)$.

${ }_{712}^{38}$ G. K. Yadava, S. Rudin, A. T. Kuhls-Gilcrist, and D. R. Bednarek, "Generalized ob713 jective performance assessment of a new high-sensitivity microangiographic fluoroscopic ${ }_{714}$ (HSMAF) imaging system," in Proc. Soc. Photo Opt. Instrum. Eng. (2008) p. 69130U.

${ }_{715}^{39}$ A. Jain, A. T. Kuhls-Gilcrist, S. K. Gupta, D. R. Bednarek, and S. Rudin, "Gener716 alized two-dimensional (2D) linear system analysis metrics (GMTF, GDQE) for digital 717 radiography systems including the effect of focal spot, magnification, scatter, and detector 718 characteristics," in Proc. SPIE Med. Imag.: Physics of Medical Imaging (2010) p. 76220K. ${ }_{719}^{40}$ P. Després, J. Rinkel, B. Hasegawa, and S. Prevrhal, "Stream processors: a new platform 720 for Monte Carlo calculations," in Journal of Physics: Conference Series, Third McGill 
721 International Workshop, Vol. 102, edited by F. Verhaegen (2008) p. 012007.

${ }^{722}{ }^{41}$ W. R. Mark, R. S. Glanville, K. Akeley, and M. J. Kilgard, "Cg: A system for pro-

723 gramming graphics hardware in a C-like language," ACM Trans. Graphics 22, 896-907 724 (2003). 